Review Paper

\title{
"Does cinema form the future of robotics?": a survey on fictional robots in sci-fi movies
}

\author{
Ehsan Saffari ${ }^{1}$ - Seyed Ramezan Hosseini ${ }^{1}$ - Alireza Taheri ${ }^{1} \cdot$ Ali Meghdari $^{1}$
}

Received: 23 September 2020 / Accepted: 11 May 2021

Published online: 22 May 2021

(c) The Author(s) 2021

OPEN

\begin{abstract}
Robotics and Artificial Intelligence (Al) have always been among the most popular topics in science fiction (sci-fi) movies. This paper endeavors to review popular movies containing Fictional Robots (FR) to extract the most common characteristics and interesting design ideas of robots portrayed in science fiction. To this end, 134 sci-fi films, including 108 unique FRs, were investigated regarding the robots' different design aspects (e.g., appearance design, interactive design and artificial intelligence, and ethical and social design). Also, in each section of this paper, some characteristics of FRs are compared with real social robots. Since some researches point to the significant role of the cinema in forming the community's expectations, it is very important to consider these characteristics and differences in choosing the future pathway of robotics. As some examples of findings, we have found that unlike the non-metallic skins/covers of real social robots, most FRs are still covered by highly detailed metal components. Moreover, the FR ability of interactions are generally (more than 90\%) shown to be similar or even more advanced than normal Human-Human interactions, and this milestone was achieved by ignoring the Al challenges of real HRI. On the other hand, the ethical aspects of movies do inspire us to consider the potential ethical aspects of real robot design. All in all, according to popularity of movies, studying FR could be a step toward more appropriate development of robotics and Al entities to be accepted by general users in the real world.
\end{abstract}

\section{Highlights:}

- We reviewed 134 sci-fi movies containing 108 unique fictional robots regarding different design aspects.

- Fictional Robot (FR) is an artificial entity acting as a result of a fictional technology and playing a role in a movie.

- Investigating fictional robots can shed light on the development of real robotics and Al entities.

Keywords Fictional robots · Science fiction movies · Social robot · Robot design · Artificial intelligence (Al) $\cdot$ Robot ethics

\section{Introduction}

After watching only a few science fiction (sci-fi) classics, one quickly realizes that robotics and artificial intelligence (Al) have always been a compelling topic in sci-fi movies.
As a manifestation of advanced technology, robots play an important role in depicting the future of humans in sci-fi movies. In fictional worlds, Fictional Robots (FR) are usually ideal candidates for unpleasant or dangerous jobs (i.e., servants, soldiers, etc.) due to their inexhaustibility,

Alireza Taheri, artaheri@sharif.edu | 'Social and Cognitive Robotics Lab., Center of Excellence in Design, Robotics, and Automation (CEDRA), Sharif University of Technology, Tehran, Iran.

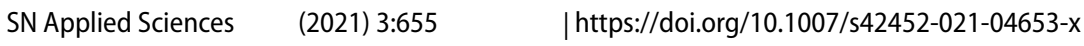


strength, and complete obedience. Moreover, robotic technology has also been used as a tool to grant human's eternal wishes: inviolable comfort and immortality.

Some researchers believe that sci-fi writers are shaping the future of technology by inspiring engineers: Marcus [1] shows some examples of technologies predicted (or inspired) by sci-fi movies; Jordan and Auernheimer [2] searched for references to Star Trek in the ACM Digital Library and found 232 papers until 2017; Some others like Jordan et al. [3] and Russell and Yarosh [4] explored the theoretical aspects of this inspiration process. Alternatively, it is also argued that these movies shape the conception of robots in ordinary people's minds, as the main potential consumers of robots in the future [5]. On the other hand Robotics and the robots' presence among the society shows an accelerating growth [6-10], especially in service positions like education $[6,7]$, health care $[8,9]$, sales and marketing [10], etc. Therefore, it is important to analyze fictional robots to better understand the market's needs and to seek inspiration for better designs. While it is no surprise that researchers are interested in studying the fiction and reality relationship, there appears to be a lack of detailed and exhaustive analysis of robotic scifi movies in the literature. Researchers have examined this relationship in different ways. Some researchers have used a limited number of movies; i.e., Lorenčík et al. [11] examined 6 sci-fi movies from many aspects such as the technology behind the stories and their real-life applications; others examined movies from a number of limited viewpoints; i.e., Bartneck [12] examined fictional robots mainly to find their attitude and similarity to humans; some other researchers studied the relationship of movies and reality in detail; i.e., Riek et al. [13] performed a trial to find a positive correlation between watching sci-fi movies and the positive attitude toward robots. Also some research groups have tried to use sci-fi materials as a tool to study robotics and $\mathrm{Al}$ as a whole; i.e., Clark $[14,15]$ analyzed the Asimov's laws of robotics for possible real-life situations. Murphy also wrote at least two books $[16,17]$ and many articles (i.e., [18-21]) to connect sci-fi movies and stories to real-life applications and challenges in robotics and Al.

The silent movie Metropolis (1927) was one of the first movies that dealt seriously with robots. Two decades later (in 1950s) Isaac Asimov published his stories which somehow organized the basic foundations of social robots [9]. Since the 1980s, advances in automation have paralleled improvements in the role of robots in cinema. With the boom of $\mathrm{Al}$ in the late twentieth century, sci-fi movies have become fascinated with the subject of robotics. In this paper, we try to examine and compare the characteristics of 108 unique fictional robots depicted in 134 of the best sci-fi movies (determined by an IMDb score above 5) through three categories: 1) Appearance design, 2) Interactive design and artificial intelligence, and 3) Ethical and social design. We endeavored to figure out the most common characteristics of fictional robots in the movies as well as the sci-fi movie makers' viewpoint regarding the robots. The situation of real social robots is also compared to the investigated fictional robots in cases where there are fundamental differences between real and fiction. To the best of our knowledge, a general survey on fictional robots or a comprehensive comparison of the orientation of fictional and real social robots has not been reported in the literature.

\section{Material and method}

In this review study, we examined 134 movies containing at least one fictional robot. Figure 1 presents the number of investigated movies with regard to their year of production and IMDb score. It should be noted that film franchises containing the same robots are considered as only one robot in this paper (i.e., the first produced movie in each series). As it is shown in Fig. 1, while there is an increasing trend in producing such movies over time, 45 movies ( 34\%) were produced in the just the last five years. Also, less than $6 \%$ of the selected movies were produced before 1980. Our consideration when selecting these movies was almost always their current popularity. Because we wanted to investigate the general expectations for future robots.

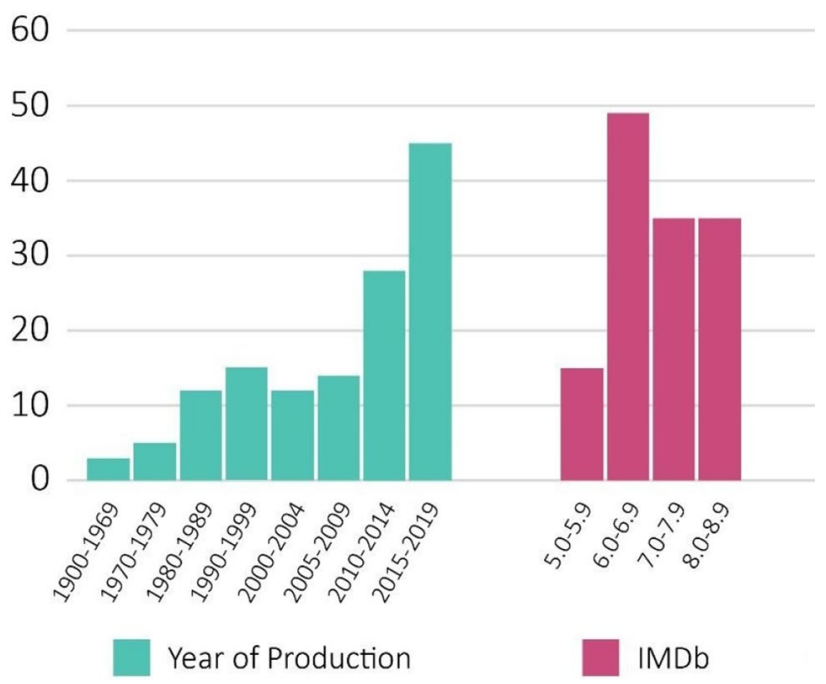

Fig. 1 Chart of the movies' year of production and IMDb score 
From these 134 movies, 108 unique fictional robots were extracted and tagged by the authors. Also, it should be mentioned that the main theme of all movies was not necessarily about robotics or Al. Therefore, we have considered any movie in which one or more fictional robots played a role. As an example, the main theme of the Star Wars series is not about robotics nor Al, but they include FRs such as "R2-D2", "C3-PO" and "BB-8". As result, in this paper, our classification is based on the number of unique fictional robots, and we ignored the number of movies in which they play.

\section{Fictional robots (FR)}

First, we propose a definition for a Fictional Robot (FR). In this paper a Fictional Robot is an artificial entity that can sense and act as a result of a (real-world or fictional) technology and played a role in at least one movie. By this definition, aliens such as "E.T." are not consider as FRs because they are not artificial entities. Also, the toy characters in the Toy Story series and characters like Pinocchio are not FRs either, because the reason behind their acting and sensing are magical and not technological (not even a fictional technology).

After identifying fictional robots, they were categorized regarding their roles, applications, and design. The fictional robots were studied in three categories: Appearance design, Artificial Intelligence and interactive design, and Ethical and social design, which are described in further detail below.

Let us classify fictional robots into seven applications and use these categories to classify FR throughout the article. Figure 2 presents the number of 108 investigated fictional robots in each category. Some robots had military or security jobs and were tagged as "Soldiers". "Caring and Entertaining robots" are classified as one group, while "Space Assistant robots" are located in another independent group. General-service robots are classified into two groups in this paper depending on if it remains as a service robot until the end of the plot or exits this duty, we refer to them as the "Servant" and "Freedman", respectively. In some other movies, robots are created to be "Immortal Humans", like "David" in A.I. (2001). Finally, the last group includes robots that are "Not Human-Made" independent creatures.

Examples for each of these 7 categories are presented below:

- Soldier: "T-1000" in Terminator 2 (1991) or "Chappie" in Chappie (2015)

- Caring \& Entertainment: "Baymax" in Big Hero 6 (2014) or "Hosts" in West World (2016)

- Space Assistant: "R2-D2" in Star Wars (1977) or "TARS" in Interstellar (2014)

- Servant: "Samanta" in Her (2013) or "Arthur" in Passengers (2016)

- Freedman: "Andrew" in Bicentennial Man (1999) or "Sonny" in I' Robot (2004)

- Immortal Humans: "Tachikoma" in Ghost in the Shell (1995) or "Alita" in Alita; Battle Angel (2019)

- Not Human-Made: Robots in Transformers (1984) or Robots (2005)

As shown in Fig. 2, fictional robots usually play the role of a service assistant or soldier (47\%). Also, in 15\% of the investigated films, robots that served as service assistants at the beginning of the story, but leave their assigned task through the main story of the film (i.e., categorized as the Freedman).
Fig. 2 The percentage of investigated movies in each fictional robots' classification category

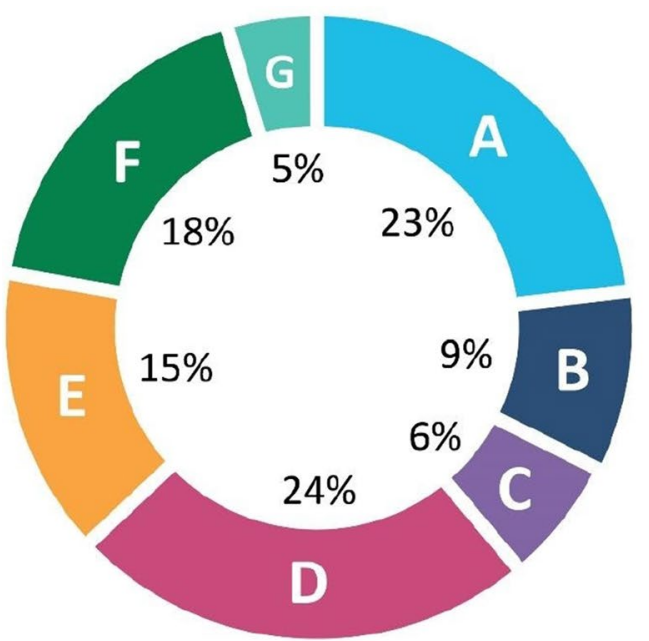




\subsection{Appearance design}

The appearance design of fictional robots was selected as an important factor to be investigated in this paper [24]. In general, the appearance design of fictional robots can be divided into the five main categories below (with some real-life robot examples):

- Human-like: Robots that are quite similar to humans in shape and look, such as Sophia, first citizen robot of the world [25].

- Metallic Humanoid: Humanoid robots with metallic parts and other obvious machine-like components, such as the Boston Dynamics' Atlas [26].

- Non-Metallic Humanoid: Humanoid robots with a nonmetallic shell, it should be noted that most real social robots are built in this way, such as the Arash Robot [27].

- Non-Humanoid: Animal-like robots and other robots with non-human-like components, such as most currently available social robots, i.e., Jibo [22] or Keepon [28].

- Non-Unique Body: Artificial intelligence entities without a single physical body, such as current voice assistants, i.e., Apple SIRI, Microsoft Cortana, etc. [29].

A diagram of the fictional robot's appearance vs. their application is presented in Fig. 3. As can be seen, most common fictional robots are Metallic Humanoid (usually representing Soldiers) and Human-like robots (usually representing Immortal Humans).
In the next section, we look closer at the FR appearance design through five different sub-categories: Material Selection, Detail Visibility, Uncanny Valley, Transformation, and Name/Gender.

\subsubsection{Material selection}

As shown in Fig. 3, most of the robots are displayed with a humanoid appearance. On the other hand, $85 \%$ (63 out of 74) of the humanoid fictional robots are created with metal. Some are displayed with a metallic appearance, and those robots which usually wear natural skins (e.g., "Mechas" in A.I. (2001)) show their metallic understructures (at least once). This fact reveals the audience's expectation of seeing robots as metallic beings. In the real world, however, the expansion of rapid prototyping technology and the cost-effectiveness of plastic has given most real social robots a plastic appearance [30-34]. Observing the function of non-metallic fictional robots (as it is shown in Fig. 3) shows that they are often used for care and entertainment purposes. For example, the "Baymax" care robot in the Big Hero 6 (2014) has an inflatable vinyl outer shell that, in addition to being flexible, enables hugging function and safe physical contact.

\subsubsection{Detail visibility}

Examining fictional robots shows that filmmakers are very interested in detailed designs, especially for metal robots. In Chappie (2015) and Robots (2005), we can see many details in the robotic character's design. Additionally,

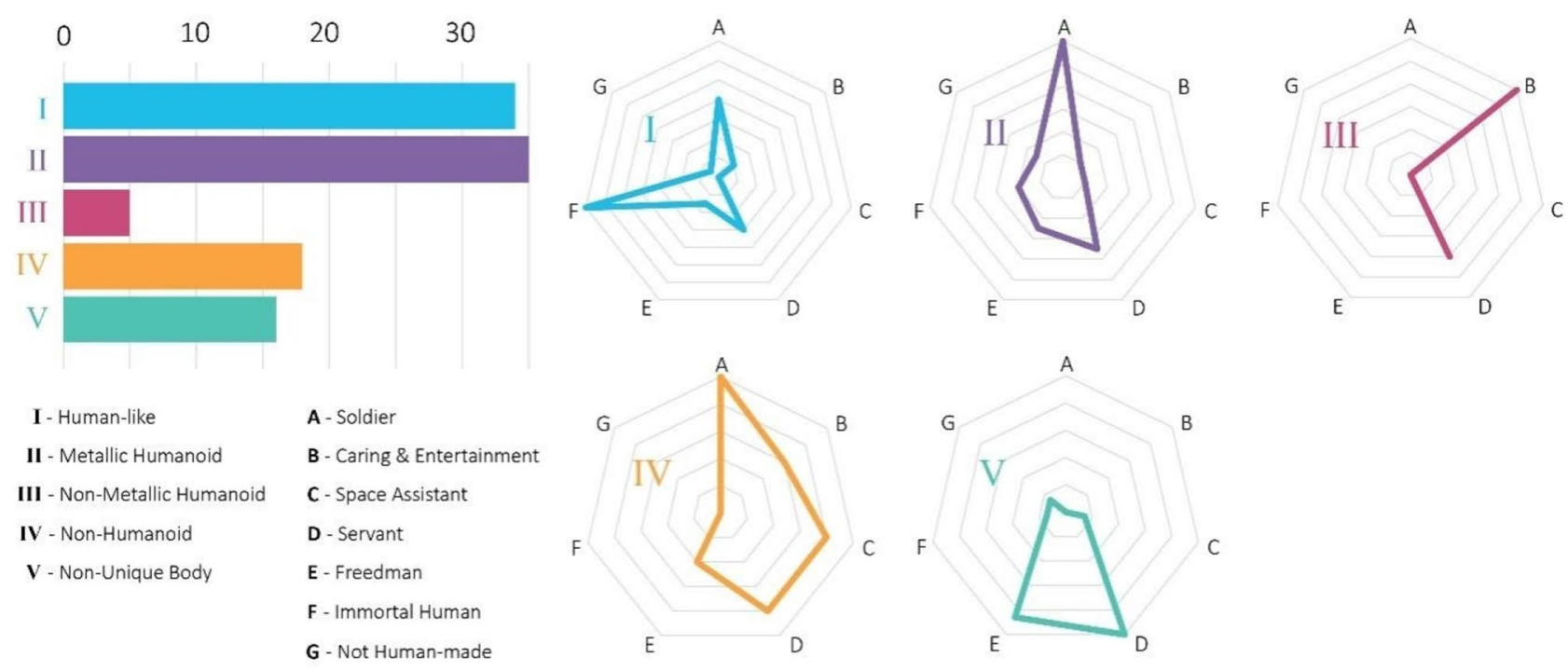

Fig. 3 Rader chart of the studied fictional robots' appearance including the number of robots and the distribution of their roles in each category 
almost all fictional humanoid robots have a mechatronic face; however, in the real world, the use of flat displays or static helmets for the robot's face is common practice $[34,35]$.

\subsubsection{Uncanny valley}

In the world of cinema, as in the real world, the Uncanny Valley phenomenon [36] is a common challenge in robots' design. Some movies (such as Uncanny (2015)) have even chosen this challenge as their main theme. When a humanoid robot's appearance is too close to a human, it causes fear and discomfort in the audience [37]. Hence, fictional humanoid robots' designers often design intentional signs in their robots so they can be easily distinguished from humans. For example, in Humans (2015-2018), this difference is the specific color of the humanoid robots' eyes (synths have shiny green eyes). An android passing as a human is one of the most common puzzles in sci-fi movies, and is often used as a surprise twist in the plot.

\subsubsection{Transformation}

One attractive feature of a fictional robot is its shape-shifting ability. This feature is so popular that the Transformers (1984-2019) franchise is entirely based on this idea. Fictional robots sometimes try to deceive their audience by changing their shape, and sometimes they use this unique feature to do a certain job or cross through specific portals.

\subsubsection{Name and gender}

As a case of anthropomorphism, the general audience tends to assign a gender to robots for better communication. This gender will be more believable with a wise choice of parameters such as appearance design, name, and tone of voice [37]. Figure 4 shows the gender distribution of the fictional robots of this study.

As can be seen in Fig. 4, approximately half of the fictional robots in the movies we studied are just male $(\sim 50 \%)$. In 12 cases, we see the robot in both genders. Also, in some cases, they are sexless, often non-humanoid robots. We observed that no female robot was portrayed as a space assistant, which may be a sign of an existing male-oriented mentality in the field.

The names chosen for fictional robots are also interesting. Examining the names shows that most fictional robots working in technical environments (in $70 \%$ of Soldiers and $100 \%$ of Space Assistants examined) have abbreviated names with numbers (such as "C3-PO" and "R2-D2" in Star Wars (1977)), while human-like robots often (in 75\% of Human-likes examined) have short, easily pronounced human names (such as "Ava" in Ex Machina (2014)). This fact is also seen in real-world robot applications [31, 34 and 35].

\subsection{Interactive design and artificial intelligence}

The robots' intelligence level and their capabilities for human interaction is an always-challenging topic in
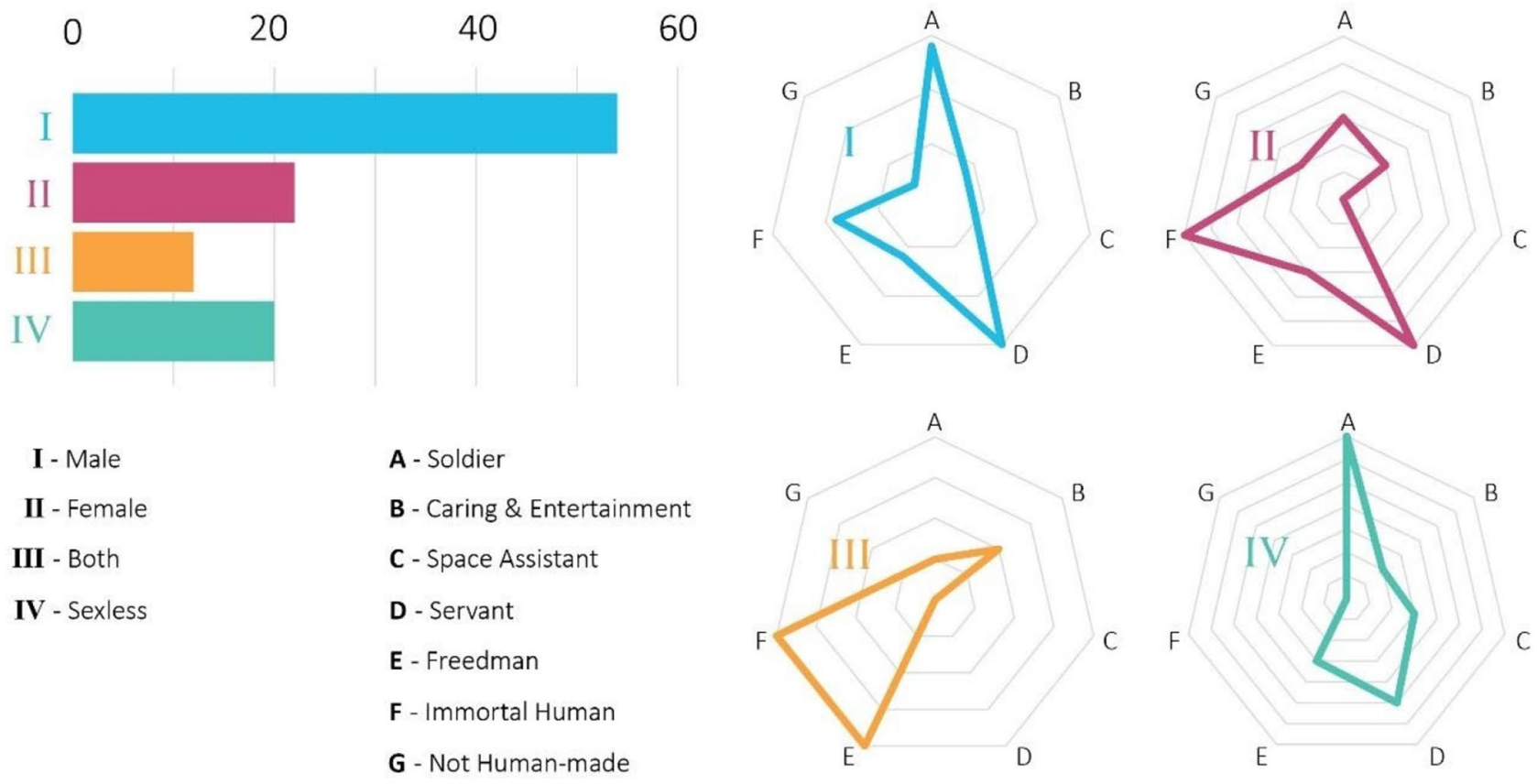

Fig. 4 Rader chart of the fictional robots' gender, including the number of robots as well as the distribution of their roles in each category 


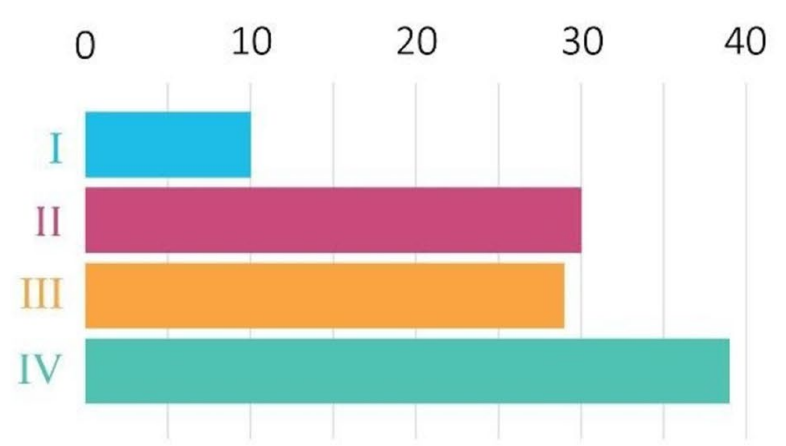

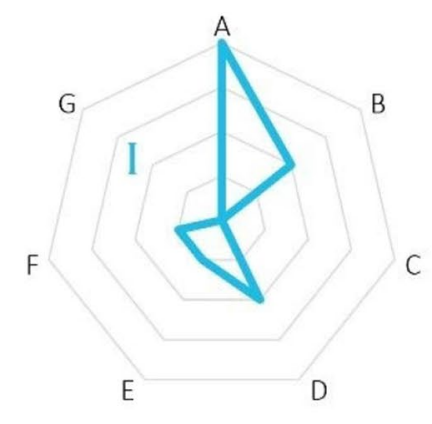
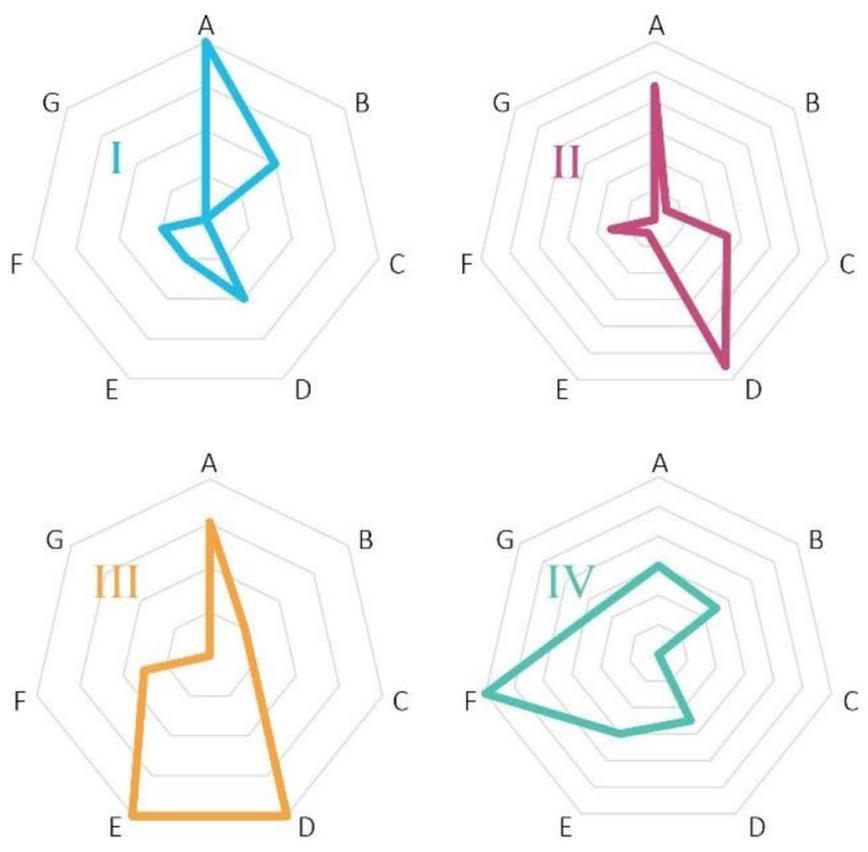
A - Soldier
B - Caring \& Entertainment
C - Space Assistant
D - Servant
E - Freedman
F - Immortal Human
G - Not Human-made

Fig. 5 Radar chart of the robots' artificial intelligence level, including the number of robots as well as the distribution of their roles in each category

fictional robots. We categorized the fictional robots into four levels in terms of artificial intelligence, including instrumental intelligence, duty intelligence, environmental intelligence, and self-awareness. These levels are further defined and described in the following section (Fig. 5).

- Instrumental Intelligence: Ability of being manually operated to quickly and accurately control actuators and the inclusion of feedback sensors,

- Duty Intelligence: Ability to carry out a mission, taking into account all environmental uncertainties,

- Environmental Intelligence: Ability to make decisions based on circumstances and conditions,

- Self-awareness: Self-awareness and self-rule.

As shown in Fig. 5, cinematographers are less interested in robots with limited levels of intelligence. This may be because the challenges of human-robot interaction, and therefore an interesting plot for a story, only arise in higher levels of artificial intelligence. In contrast to fiction, most real robots are still limited to instrumental intelligence, and many technical challenges remain in areas such as reliability, accuracy, etc. [34, 38]. In the following section, we studied fictional robots in four subsections referred to as Human-like Interactivity, Superhuman Interactivity, Fictional Al, and Cyborgs.

\subsubsection{Human-like interactivity}

In order for robots to be able to live among humans, they need to adapt to human living conditions and be able to interact with humans. Fictional robots are no exception to this rule. Hence, one of the obvious characteristics of humanoid robots in most movies is the obvious/definite ability to establish human-like interactions. Of the 74 fictional humanoid robots examined, 68 (i.e., $92 \%$ ) had the ability to interact with humans at an almost human level. From this we predict that after observing the humanoid appearance and skill of these fictional robots, the general public has come to expect fully human-like interaction ability from future robots. It should be noted that this prediction needs more surveys by the help of questionnaire analysis.

\subsubsection{Superhuman interactivity}

Many fictional robots can be distinguished from humans because of their advanced sensory capabilities, which we refer to as "superhuman interactivity". Sci-fi writers often try to make their fictional robots superior to humans by giving them special sensory-motor abilities. The most common superhuman interactivity (found in 42 of the observed movies) is advanced image processing, such as 
accurate lip-reading ("HAL" in 2001: A Space Odyssey (1968)) or sophisticated online processing (fast medical scanning of "Baymax" in Big Hero 6 (2014)).

\subsubsection{Fictional Al}

By investigating the characters of artificial intelligence in science fiction films, we can conclude that verbal interaction (i.e., the recognition and synthesis of speech) is considered a necessary ability for an Al entity. Moreover, the entity's ability to speak gives it an identity and personality, and processing the correct speech tone makes it sound commanded and obedient. This is in line with the importance of natural language processing in real-life artificial intelligence reported in [39].

Another human-like ability the audience expects to see from an Al entity is its ability to learn. An advanced fictional Al entity can easily learn new concepts and topics and improve themselves [15]. The subject of robot learning has also received a great deal of research attention in recent years, and several methods have been developed to achieve this ability for robots/machines [40-42].

Moreover, although Humans are limited in terms of information processing and storage, the fictional robot's access to information (using the Internet or even other fictional networks) is almost unlimited, and in some cases, robots are given unlimited control of other digital entities. This feature is more common in bodiless robots. For example, in I, Robot (2004), a central AI called "VIKI" with the full control of all other robots is trying to take over the city.

In addition, sci-fi movies rarely try to address real-life engineering challenges, such as the memory and information management challenges found in real-world robots [43]; however, in few movies, such as the Next Gen (2018) animation, this issue is observed when the robot is forced to erase his memories due to memory overload.

\subsubsection{Cyborgs}

Alongside robots, fictional cyborgs also play a role in some sci-fi movies. A cyborg is a human with attached robotic plugins or limbs, or at its most radical level, a human brain controlling a whole robotic body. In our movie database, 27 out of 134 movies (i.e., 12\%) addressed cyborgs. Among them, RoboCop (1987) and Alita: Battle Angel (2019) are widely known examples.

In movies and stories, cyborgs are the best form to address the centuries-old theme of the clash of human nature and machinery. Although connecting a human brain to a robotic body or artificial limbs to a human is an extremely challenging process with our current knowledge and medical limitations, sci-fi writers consider it common practice and portray cyborgs as the perfect examples of this clash of humanity and machinery (i.e., Ghost in the Shell (1995)).

\subsection{Ethical and social design}

Today, with the rapid expansion of artificial intelligence and social robotics $[31,44]$, the issue of the ethical and social design of robots has become very important [27]. In recent years, several articles have been published by psychologists and sociologists studying the ethical standards and issues in robot design [23]. Similarly, sci-fi writers have shown a great deal of concern for the potential moral challenges of advanced robotics, especially from a human-robot interaction perspective. Also, they may employ fictional robots as metaphorical tools to discuss human ethical challenges. Several of the most important ethical features observed in fictional robots (including Desire for Survival and Evolution, Loyalty, Emotions and Personality, and Robot Abuse) are presented in the following section. Of course, different movies approach these areas with different and diverse viewpoints, i.e. in some movies robots/humans are seeking eternal life while in others they refuse eternal life for different reasons (such as Andrew's rejection of immortal life in Bicentennial Man (1999)).

\subsubsection{Desire for survival and evolution}

One can claim that the first goal of any living creature is to survive. Fictional robots, when they reach the level of environmental intelligence or self-awareness, consider themselves living beings, and this desire to survive also arises in them. The desire to survive leads the robot to begin to experience emotions such as fear, and the desire for evolution leads to the emotion of greed. For example, in A.I. (2001) and Her (2013), we see a desire for survival and evolution, respectively. These tendencies can cause war or serious conflict between humans and robots when an artificial intelligence entity, sees the destruction of humans (certain humans or humanity as a whole) as the first step of robots' survival or evolution, i.e., 2001: A Space Odyssey (1968) and I, Robot (2004), respectively.

Although the audience expects a self-aware robot to have a desire for survival and evolution, having creativity and the ability to dream is not expected from a robot. Hence, this occurrence could be surprising in movies like Chappie (2015) and I, Robot (2004). 


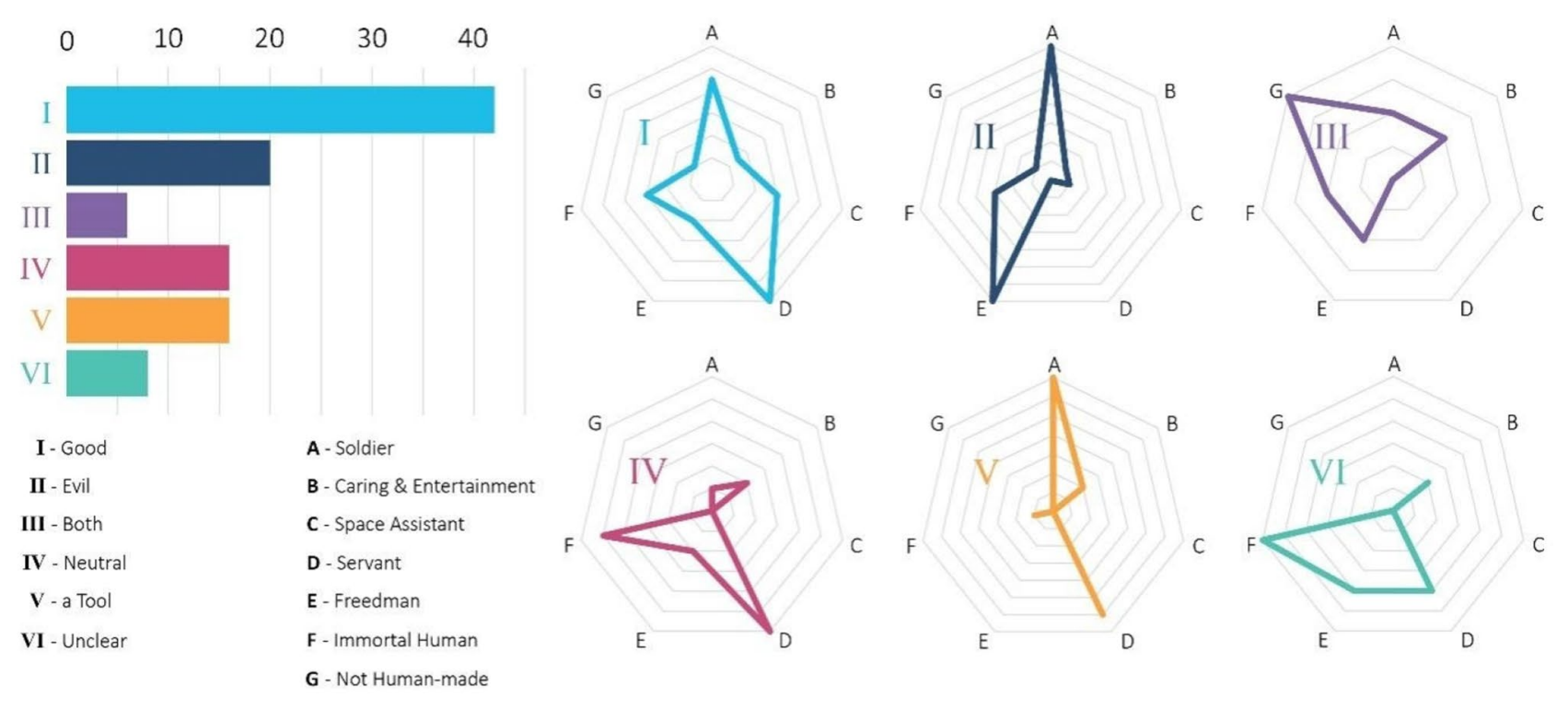

Fig. 6 Radar chart of the robot's overall behaviors, including the number of robots as well as the distribution of their roles in each category

\subsubsection{Loyalty}

According to our review, 49 out of the 59 fictional robots that play a servant or assistant role showed considerable loyalty to their owners and only ten servant robots violated this loyalty. In some cases, this is due to their limited task intelligence, but sometimes it is due to their ingenuity. For example, the fidelity of the robot in Robot \& Frank (2012) is due to its conscientiousness, while the fictional robot in The Iron Giant (1999) learns to be loyal after befriending humans.

Also, the audience usually expects robots to be obedient, lawful, and insensitive entities. Also, a loyal robot must be value-oriented (i.e., Chappie (2015)) and self-sacrificing (i.e., The Iron Giant (1999)).

Alternatively, one can categorize fictional robots according to their overall behaviors (e.g., good, evil, both, neutral, and a tool). Figure 6 presents the distribution of the robot's overall behavior in the investigated dataset. It should be noted that we could not classify the robots' behaviors for $8 \mathrm{FRs}$ and use the label "unclear" in for them Fig. 6.

\subsubsection{Emotions and personality}

If we accept that being able to act and express feelings, through changes in facial expressions, a change in tone of voice, and so on, means a robot has real feelings, we can then claim that the imagination of sci-fi writers has come true. It is also obvious that many human personality traits can be loaded into the robot's artificial intelligence in the form of computer codes [45]. This kind of coding was seen abundantly in the reviewed films. In some movies, such as Eva (2011) and Interstellar (2014), we see the ability to customize the robot's emotions and personality parameters, a feature now seen in real social robots such as KIKI [46].

Moreover, in the reviewed movies, there is a strong relationship between having memory and having human-like emotions, including memory retrieval helping robots to have deeper and stronger emotions (i.e., The Iron Giant (1999), losing memory being equal to losing personality and emotions (i.e., Wall-E (2008)), and injecting human memories into a robot causing it to have the same emotions (i.e., Astro Boy (2009) and West World (2016-2020)), etc. These observations are in line with real-life studies on long-term human-robot interactions in which having long-term emotional relations and personality is linked to having a good memory and remembering their shared memories in subsequent encounters $[47,48]$. This type of capability is provided in a limited version in the real social robots like Cozmo [49].

An important lesson to learn from these movies when designing a robots' emotion and personality, is that although a robot's feelings and personality is artificial, a person's feelings toward a robot can be quite real (Her (2013)). This issue is a main theme in movies which include fictional robots as adopted children or lovers, such as A.I. (2001) and Eva (2011).Interestingly, de Graaf [50] also claimed that human-robot interactions are constructed based on the human-human interaction rules. 


\subsubsection{Robot abuse}

Most fictional robots are depicted as man-made creatures, and therefore, are considered inferior to their human creators [51]. As an immediate result of this unique relationship, humans feel a mixture of feelings toward their creations: superiority, love, fear, pity, etc. Some, as a result of negative emotions behave tyrannical, indifferent, and sometimes abusive toward their own creatures [52-54]. Examples of this kind of cruel behavior are depicted in movies such as A.I. (2001) and Humans series (2015-2018).

This fact has led some screenwriters to employ these believable but exaggerated examples as metaphors to study issues such as racism, xenophobia, and class divisions in human society. It can be seen in the literature that humans like to have control over robots and they reject dominant behavior of robots $[55,56]$. This phenomenon can be seen in movies such as I am Mother (2019) or Tau (2018). Therefore, we see a common theme of robot abuse and robophobia in sci-fi literature and movies (which may result in a growth of these negative feelings among their audience). Just as in human history, one observes rebellion, conspiracy, and open war in response to this discrimination as some selfconscious robots try to overcome their creators by seeking freedom, independence, and equal rights. The West World series (2016-2020) is one of the most recent examples of this theme.

\section{Discussion}

After discussing fictional robots from three perspectives: appearance design, interactive design and artificial intelligence, and ethical and social design, it is necessary to draw some general conclusions from the topic. We hope that these issues and all the issues raised so far in this article will be a step toward better development of robotics and artificial intelligence systems in the real world.

\subsection{Thirst for power}

Man has always sought to create tools for perfection and immortality, and the robot is another one of these. As we can see in movies such as Iron Man (2008) or RoboCop (1987), there is an assumption that robotic technology will be able to turn ordinary or even disabled persons into superheroes with superhuman powers. Conversely, some movies often warn people of their excessive desire for power, which can sometimes backfire and destroy already achieved goals.

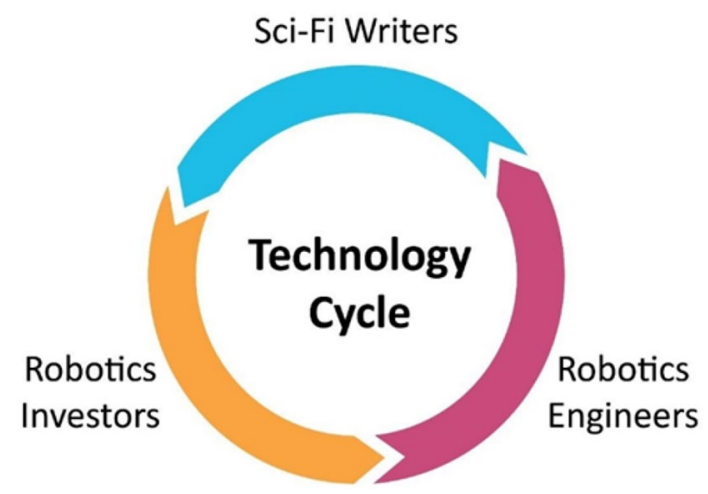

Fig. 7 Technology cycle chart

\subsection{Fiction vs. reality}

Although fictional robots are "built" on scientific foundations/principles, some capabilities (like great ability in natural language processing) found in a fictional robot created half a century ago are still unattainable in today's real robots. Nevertheless, it is an assumption that fictional robots have inspired the development of technology and the creation of real robots, especially the social robots. As evidence to this claim, we can compare the sound of the R2-D2 robot vs. the Cozmo robot [49], the name and function of the Weebo robot vs. the Jibo robot [22], and the prototype toys of the BB-8 [57] and DIY implementations of R2-D2 [58].

\subsection{Technology cycle}

The increasing number of fictional robots in recent years shows the great interest of screenwriters and audiences in this technology. As sci-fi writers shape the audience's expectations of the robot [5], they can inspire robotics policymakers and investors, who turn to engineers to bring these fantasies closer to reality. As technology takes another step further, this again inspires writers to find/create new ideas for their stories and screenplays. Therefore, fictional robots play an important role in the development of robotic technology by creating a selfperpetuating technology cycle, Fig. 7.

As an example, during a visit to Carnegie Mellon University's Robotics Laboratory, Don Hall was inspired to create Baymax (The robot in Big Hero 6 (2014)). After making the film, Dr. Christopher G. Atkeson, a professor at that robotics lab, decided to make a real Baymax robot and defined a new field of projects called inflatable robotics. "It (Big Hero 6 (2014)) is a tremendous win for soft robotics," says Dr. Atkeson [59]. 


\section{Conclusion}

Movies containing fictional robots are among the most popular films in today's cinema. In this article, we reviewed 134 well-known sci-fi movies containing 108 unique fictional robots and categorized the characteristics and most common points of the robots based on different aspects of robot design. It was observed that the preferred material for fabricating fictional robots are metals with lots of detail components contrary to the commonly used plastic materials used in real social robots' design. In addition, we indicated that unlike the existing challenge to empower Al robots to behave naturally in real HRI, fictional robots are usually able to interact with humans in an advanced, almost human-like way. We believe that the robotics' community can be inspired by the ethical challenges portrayed in sci-fi films to consider ethical aspects when designing real social robots. Investigating fictional robots can shed light on the development of real robotics and Al entities, even though an enormous gap exists between the fictional and real robots' design and capabilities.

The main limitation of the current study is in the movie selection stage, nearly all of the movies studied in this paper were in English (with few exceptions) and most were produced in the USA and Western Europe. Therefore, we have missed the opportunity to study Non-Western perspectives toward robots and Al in society. In particular, the absence of Japanese sci-fi anime, as a resource full of different aspects of technologies, is a huge drawback. Another limitation of this paper was restricting the study to movies. Extending the domain of study to sci-fi literature will lead to a more diverse and rich study with a greatly extended time frame.

Acknowledgements We appreciate the Dr. AliAkbar Siassi Memorial Grant for the complementary support of the Social \& Cognitive Robotics Laboratory.

Author contributions All authors contributed equally to the manuscript.

Declarations

Conflict of interest All authors Ehsan Saffari, Seyed Ramezan Hosseini, Alireza Taheri, and Ali Meghdari declare that they have no conflict of interest.

Open Access This article is licensed under a Creative Commons Attribution 4.0 International License, which permits use, sharing, adaptation, distribution and reproduction in any medium or format, as long as you give appropriate credit to the original author(s) and the source, provide a link to the Creative Commons licence, and indicate if changes were made. The images or other third party material in this article are included in the article's Creative Commons licence, unless indicated otherwise in a credit line to the material. If material is not included in the article's Creative Commons licence and your intended use is not permitted by statutory regulation or exceeds the permitted use, you will need to obtain permission directly from the copyright holder. To view a copy of this licence, visit http://creativecommons. org/licenses/by/4.0/.

\section{Appendix}

See Table 1 
Table 1 List of the Investigated Fictional Robots including the movie's name they present and the year of first presence

\begin{tabular}{|c|c|c|c|c|c|c|c|}
\hline & FR Name & Movie Name & Year & & FR Name & Movie Name & Year \\
\hline 1 & Maria & Metropolis & 1927 & 55 & David & Prometheus & 2012 \\
\hline 2 & Robby & Forbidden Planet & 1956 & 56 & VGC-60L & Robot and Frank & 2012 \\
\hline 3 & HAL & 2001: A Space Odyssey & 1968 & 57 & Soldiers & Total Recall & 2012 \\
\hline 4 & Hosts & West World & 1973 & 58 & Dorian (DRN) & Almost Human & 2013 \\
\hline 5 & Wives & The Stepford Wives & 1975 & 59 & MX-43 & Almost Human & 2013 \\
\hline 6 & Proteus IV & Demon Seed & 1977 & 60 & Ash & Black Mirror: Be Right Back & 2013 \\
\hline 7 & $\mathrm{C}-3 \mathrm{PO}$ & Star Wars & 1977 & 61 & Soldiers & Elysium & 2013 \\
\hline 8 & R2-D2 & Star Wars & 1977 & 62 & Samantha & Her & 2013 \\
\hline 9 & Ash & Alien & 1979 & 63 & Kelex, Kelor & Man of Steel & 2013 \\
\hline 10 & Replicates & Blade Runner & 1982 & 64 & Tet & Oblivion & 2013 \\
\hline 11 & Gadget & Inspector Gadget & 1983 & 65 & Jaegers & Pacific Rim & 2013 \\
\hline 12 & Edgar & Electric Dreams & 1984 & 66 & Ava (Machine) & The Machine & 2013 \\
\hline 13 & $\mathrm{~T}-800$ & The Terminator & 1984 & 67 & Pilgrims & Automata & 2014 \\
\hline 14 & Transformers & Transformers & 1984 & 68 & Baymax & Big Hero 6 & 2014 \\
\hline 15 & Daryl & D.A.R.Y.L & 1985 & 69 & Ava & Ex Machina & 2014 \\
\hline 16 & Johnny 5 & Short Circuit & 1986 & 70 & TARS, CASE, PLEX, KIPP & Interstellar & 2014 \\
\hline 17 & RoboCop & RoboCop & 1987 & 71 & Soldiers & RoboCop & 2014 \\
\hline 18 & -- & Hardware & 1990 & 72 & -- & The Nostalgist & 2014 \\
\hline 19 & Taxi Driver & Total Recall & 1990 & 73 & Dr. Will Caster & Transcendence & 2014 \\
\hline 20 & $\mathrm{~T}-1000$ & $\begin{array}{l}\text { Terminator 2: Judgment } \\
\text { Day }\end{array}$ & 1991 & 74 & F.R.I.D.A.Y & Avengers: Age of Ultron & 2015 \\
\hline 21 & The Dinosaur & Redhat and Cousin & 1994 & 75 & Chappie & Chappie & 2015 \\
\hline 22 & Tachikoma & Ghost in the Shell & 1995 & 76 & Moose & Chappie & 2015 \\
\hline 23 & Data & Star Trek: First Contact & 1996 & 77 & Synth & Humans & 2015 \\
\hline 24 & Weebo & Flubber & 1997 & 78 & BB-8 & $\begin{array}{l}\text { Star Wars: The Force } \\
\text { Awakens }\end{array}$ & 2015 \\
\hline 25 & NDR-114 & Bicentennial Man & 1999 & 79 & T-3000 & Terminator: Genisys & 2015 \\
\hline 26 & Bender Bending Rodríguez & Futurama & 1999 & 80 & Athena & Tomorrowland & 2015 \\
\hline 27 & The Iron Giant & The Iron Giant & 1999 & 81 & -- & Uncanny & 2015 \\
\hline 28 & Sentinel & The Matrix & 1999 & 82 & Amelia & Amelia 2.0 & 2016 \\
\hline 29 & David & $\mathrm{Al}$ & 2001 & 83 & ADls; Robotic Bees & $\begin{array}{l}\text { Black Mirror: Hated in the } \\
\text { Nation }\end{array}$ & 2016 \\
\hline 30 & Mechas & $\mathrm{Al}$ & 2001 & 84 & Howard & Infinity Chamber & 2016 \\
\hline 31 & Teddy & $\mathrm{Al}$ & 2001 & 85 & S.A.R & Kill Command & 2016 \\
\hline 32 & Red Queen & Resident Evil & 2002 & 86 & Arthur & Passengers & 2016 \\
\hline 33 & B.E.N & Treasure Planet & 2002 & 87 & $\mathrm{~K}-2 \mathrm{SO}$ & $\begin{array}{l}\text { Rogue One: A Star Wars } \\
\text { Story }\end{array}$ & 2016 \\
\hline 34 & $T-X$ & $\begin{array}{l}\text { Terminator: Rise of the } \\
\text { Machines }\end{array}$ & 2003 & 88 & Hosts & West World & 2016 \\
\hline 35 & Briareos & Appleseed & 2004 & 89 & Robotic Dogs & Black Mirror: Metalhead & 2017 \\
\hline 36 & Sonny & I, Robot & 2004 & 90 & Karen; Spider Man Cloth & Spider-Man: Homecoming & 2017 \\
\hline 37 & VIKI & I, Robot & 2004 & 91 & The Dog & A.X.L & 2018 \\
\hline 38 & Dr. Otto Octavius & Spider-Man 2 & 2004 & 92 & Synths & Extinction & 2018 \\
\hline 39 & Robots & Robots & 2005 & 93 & Gen 6 & Next Gen & 2018 \\
\hline 40 & Marvin & $\begin{array}{l}\text { The Hitchhiker's Guide to } \\
\text { the Galaxy }\end{array}$ & 2005 & 94 & Project 77 & Next Gen & 2018 \\
\hline 41 & ARIIA & Eagle Eye & 2008 & 95 & Replicas & Replicas & 2018 \\
\hline 42 & Iron Man & Iron Man & 2008 & 96 & Tau & Tau & 2018 \\
\hline 43 & JARVIS & Iron Man & 2008 & 97 & STEM & Upgrade & 2018 \\
\hline 44 & Gerty & Moon & 2008 & 98 & Synths & Zoe & 2018 \\
\hline 45 & Astro Boy & Astro Boy & 2009 & 99 & Alita & Alita; Battle Angel & 2019 \\
\hline
\end{tabular}


Table 1 (continued)

\begin{tabular}{|c|c|c|c|c|c|c|c|}
\hline & FR Name & Movie Name & Year & & FR Name & Movie Name & Year \\
\hline 46 & Surrogates & Surrogates & 2009 & 100 & Ashley Too & $\begin{array}{l}\text { Black Mirror: Rachel, Jack } \\
\text { and Ashley Too }\end{array}$ & 2019 \\
\hline 47 & Skynet (T-5000) & Terminator: Salvation & 2009 & 101 & Soldiers & Code 8 & 2019 \\
\hline 48 & Eve & Wall.E & 2009 & 102 & The Mother & I am Mother & 2019 \\
\hline 49 & Wall.E & Wall.E & 2009 & 103 & -- & $\begin{array}{l}\text { Love, Death \& Robots: } \\
\text { Blindspot }\end{array}$ & 2019 \\
\hline 50 & Eva & Eva & 2011 & 104 & Yan & $\begin{array}{l}\text { Love, Death \& Robots: } \\
\text { Good Hunting }\end{array}$ & 2019 \\
\hline 51 & Gris; the Cat & Eva & 2011 & 105 & Agriculture Machines & Love, Death \& Robots: Suits & 2019 \\
\hline 52 & Max & Eva & 2011 & 106 & $\begin{array}{l}\text { K-VRC, XBOT 4000, Triangu- } \\
\text { lar bot }\end{array}$ & $\begin{array}{l}\text { Love, Death \& Robots: } \\
\text { Three Robots }\end{array}$ & 2019 \\
\hline 53 & Prototype 519 (SI-9) & Eva & 2011 & 107 & Zima Blue & $\begin{array}{l}\text { Love, Death \& Robots: Zima } \\
\text { Blue }\end{array}$ & 2019 \\
\hline 54 & Atom & Real Steel & 2011 & 108 & Rev-9 & Terminator: Dark Fate & 2019 \\
\hline
\end{tabular}

\section{References}

1. Marcus A (2013) The history of the future: sci-fi movies and $\mathrm{HCl}$. Interactions 20(4):64-67

2. Jordan P, Auernheimer B (2017) The fiction in computer science: a qualitative data analysis of the ACM digital library for traces of star trek. In: International conference on applied human factors and ergonomics, pp 508-520. Springer, Cham

3. Jordan P, Mubin O, Silva PA (2016) A conceptual research agenda and quantification framework for the relationship between science-fiction media and human-computer interaction. In: International conference on human-computer interaction, pp 52-57. Springer, Cham

4. Russell DM, Yarosh S (2018) Can we look to science fiction for innovation in $\mathrm{HCl}$ ?. Interactions, 25(2), 36-40

5. Kriz S, Ferro TD, Damera P, Porter JR (2010) Fictional robots as a data source in HRI research: Exploring the link between science fiction and interactional expectations. In: 19th international symposium in robot and human interactive communication, pp 458-463. IEEE

6. Alemi M, Meghdari A, Ghazisaedy M (2015) The impact of social robotics on L2 learners' anxiety and attitude in English vocabulary acquisition. Int J Soc Robot 7(4):523-535

7. Shahab M, Taheri A, Hosseini SR, Mokhtari M, Meghdari A, Alemi M, Pouretemad H, Shariati A, Pour AG (2017, October). Social Virtual reality robot (V2R): a novel concept for education and rehabilitation of children with autism. In: 2017 5th RSI International Conference on Robotics and Mechatronics (ICROM). IEEE, pp 82-87

8. Meghdari A, Shariati A, Alemi M, Nobaveh AA, Khamooshi M, Mozaffari $B$ (2018) Design performance characteristics of a social robot companion "Arash" for pediatric hospitals. Int J Humanoid Rob 15(05):1850019

9. Meghdari A, Alemi M, Zakipour M, Kashanian SA (2019) Design and realization of a sign language educational humanoid robot. J Intell Rob Syst 95(1):3-17

10. Zibafar A, Saffari E, Alemi M, Meghdari A, Faryan L, Pour AG, RezaSoltani A, Taheri A (2019) State-of-the-art visual merchandising using a fashionable social robot: RoMa. Int J Soc Robot, 1-15

11. Lorenčík $D$, Tarhaničová $M$, Sinčák $P$ (2013) Influence of sci-fi films on artificial intelligence and vice-versa. In: 2013 IEEE 11th international symposium on applied machine intelligence and informatics (SAMI) pp 27-31. IEEE
12. Bartneck $C$ (2013) Robots in the theatre and the media

13. Riek LD, Adams A, Robinson P (2011) Exposure to cinematic depictions of robots and attitudes towards them. In: Proceedings of International Conference on Human-Robot Interaction, Workshop on Expectations and Intuitive Human-Robot Interaction

14. Clarke R (1993) Asimov's laws of robotics: implications for information technology-Part I. Computer 26(12):53-61

15. Clarke R (2011) Asimov's laws of robotics: Implications for information technology. Machine ethics, 254-84

16. Murphy RR (2019) Learn Al and Human-Robot Interaction from Asimov's I, Robot Stories: Robotics Through Science Fiction vol. 2.

17. Murphy RR (ed) (2018) Robotics Through Science Fiction: Artificial Intelligence Explained Through Six Classic Robot Short Stories. MIT Press

18. Murphy RR (2018) Pacific Rim and exoskeletons. Sci Robotics, 3(16)

19. Murphy RR(2018) Westworld and the uncanny valley. Sci Robotics, 3(17)

20. Murphy RR (2018) Meet L3-37, an elite self-modifying robot in Solo: A Star Wars Movie. Sci Robotics, 3(19)

21. Murphy RR (2019) The real Alita: Battle Angel cyborgs. Sci Robotics, 4(27).

22. Breazeal CL (2014) JIBO, The World's First Social Robot for the Home. Indiegogo. Available online at https://www.indiegogo. com/projects/jibo-the-world-s-first-socialrobot-for-the-home, checked on, 1(22), 2019

23. Allen C, Wallach W, Hughes JJ, Bringsjord S, Taylor J, Sharkey N, O'Meara R (2011) Robot ethics: the ethical and social implications of robotics. MIT press

24. Strait MK, Aguillon C, Contreras V, Garcia N (2017) The public's perception of humanlike robots: Online social commentary reflects an appearance-based uncanny valley, a general fear of a "Technology Takeover", and the unabashed sexualization of female-gendered robots. In: 2017 26th IEEE International Symposium on Robot and Human Interactive Communication (ROMAN) pp 1418-1423. IEEE.

25. Retto J (2017) Sophia, first citizen robot of the world. ResearchGate https://www.researchgate. net, 2-9

26. Atlas. Boston Dynamics. Available online at https://www.bosto ndynamics.com/atlas, checked on, 5(8), 2020 
27. Meghdari A, Shariati A, Alemi M, Vossoughi GR, Eydi A, Ahmadi E, Mozafari B, Amoozandeh Nobaveh A, Tahami R (2018) Arash: a social robot buddy to support children with cancer in a hospital environment. Proc Inst Mech Eng [H] 232(6):605-618

28. Kozima H, Michalowski MP, Nakagawa C (2009) Keepon. Int J Soc Robot 1(1):3-18

29. Hoy MB (2018) Alexa, Siri, Cortana, and more: an introduction to voice assistants. Med Ref Serv Q 37(1):81-88

30. Broekens J, Heerink M, Rosendal H (2009) Assistive social robots in elderly care: a review. Gerontechnology 8(2):94-103

31. Belpaeme T, Kennedy J, Ramachandran A, Scassellati B, Tanaka F (2018) Social robots for education: A review. Sci Robotics 3(21), eaat5954

32. Sim DYY, Loo CK (2015) Extensive assessment and evaluation methodologies on assistive social robots for modelling humanrobot interaction-a review. Inf Sci 301:305-344

33. van den Berghe R, Verhagen J, Oudgenoeg-Paz O, van der Ven $\mathrm{S}$, Leseman P (2019) Social robots for language learning: a review. Rev Educ Res 89(2):259-295

34. Scassellati B, Admoni H, Matarić M (2012) Robots for use in autism research. Annu Rev Biomed Eng 14:275-294

35. Mokhtari $M$, (2019) Designing a robot head for studying social interaction with the ability to express emotions using a projector (in Persian), Master Thesis, Sharif University of Technology, Tehran, Iran, 9-10

36. Mori M (1970) The uncanny valley. Energy 7(4):33-35

37. Alesich S, Rigby M (2017) Gendered robots: implications for our humanoid future. IEEE Technol Soc Mag 36(2):50-59

38. Skinner J, Hall D, Zhang H, Dayoub F, Sünderhauf N (2019) The probabilistic object detection challenge. arXiv preprint arXiv: 1903.07840

39. Saffari E, Meghdari A, Vazirnezhad B, Alemi M (2015) Ava (a social robot): Design and performance of a robotic hearing apparatus. In: International Conference on Social Robotics, pp 440-450, Springer, Cham

40. Ramírez OAl, Khambhaita $\mathrm{H}$, Chatila $\mathrm{R}$, Chetouani M, Alami R (2016) Robots learning how and where to approach people. In: 2016 25th IEEE international symposium on robot and human interactive communication (RO-MAN), pp 347-353. IEEE

41. Rahmatizadeh R, Abolghasemi P, Bölöni L, Levine S (2018) Vision-based multi-task manipulation for inexpensive robots using end-to-end learning from demonstration. In: 2018 ieee international conference on robotics and automation (ICRA) (pp. 3758-3765). IEEE.

42. Zhu Z, Hu H (2018) Robot learning from demonstration in robotic assembly: a survey. Robotics $7(2): 17$

43. Li F, Yang S, Yi X, Yang X (2017) Towards visual SLAM with memory management for large-scale environments. In: Pacific Rim Conference on Multimedia, pp 776-786. Springer, Cham
44. Taheri A, Meghdari A, Alemi M, Pouretemad H (2018) Humanrobot interaction in autism treatment: a case study on three pairs of autistic children as twins, siblings, and classmates. Int J Soc Robot 10(1):93-113

45. Pour AG, Taheri A, Alemi M, Meghdari A (2018) Human-robot facial expression reciprocal interaction platform: case studies on children with autism. Int J Soc Robot 10(2):179-198

46. KIKI Robot. Zoetic AI Official Website. Available online at https:// www.kiki.ai, checked on, 5(8), 2020

47. Baxter $P$, Belpaeme $T$, CanameroL, Cosi $P$, Demiris $Y$ Enescu $V$ (2011) Long-term human-robot interaction with young users. In: IEEE/ACM human-robot interaction 2011 conference (robots with children workshop) (Vol. 80)

48. Kasap Z, Magnenat-Thalmann N (2012) Building long-term relationships with virtual and robotic characters: the role of remembering. Vis Comput 28(1):87-97

49. Meet Cozmo. Anki-US. Available online at https://www.anki. com/en-us/cozmo.html, checked on, 5(8), 2020.

50. de Graaf MM (2016) An ethical evaluation of human-robot relationships. Int J Soc Robot 8(4):589-598

51. Kim MS, Kim EJ (2013) Humanoid robots as "The Cultural Other": are we able to love our creations? Al \& Soc 28(3):309-318

52. Whitby B (2008) Sometimes it's hard to be a robot: A call for action on the ethics of abusing artificial agents. Interact Comput 20(3):326-333

53. Nomura T, Kanda T, Kidokoro H, Suehiro Y, Yamada S (2016) Why do children abuse robots? Interact Stud 17(3):347-369

54. Bartneck C, Rosalia C, Menges R, Deckers I (2005) Robot abuse-a limitation of the media equation

55. Jarrasse N, Sanguineti V, Burdet E (2014) Slaves no longer: review on role assignment for human-robot joint motor action. Adapt Behav 22(1):70-82

56. Reinhardt J, Pereira A, Beckert D, Bengler K (2017) Dominance and movement cues of robot motion: A user study on trust and predictability. In: 2017 IEEE international conference on systems, man, and cybernetics (SMC), pp 1493-1498. IEEE

57. Kevin McFarland. The Story (And Tech) Behind That Awesome Star Wars BB-8 Toy. WIRED. Available online at wired. com/2015/09/bb8-the-inside-story/, checked on, 5(8), 2020

58. Paul Gentile. The Comprehensive Guide to Building a Realistic R2-D2 Replica. Makezine. Available online at https://makezine. com/projects/building-your-first-r2/, checked on, 12(13), 2020

59. https://www.cmu.edu/news/stories/archives/2014/october/ october29_baymax.html (May 2020)

Publisher's Note Springer Nature remains neutral with regard to jurisdictional claims in published maps and institutional affiliations. 